\title{
APPROXIMATELY BISECTRIX-ORTHOGONALITY PRESERVING MAPPINGS
}

\author{
ALI ZAMANI
}

\begin{abstract}
Regarding the geometry of a real normed space $\mathcal{X}$, we mainly introduce a notion of approximate bisectrix-orthogonality on vectors $x, y \in \mathcal{X}$ as follows:

$$
x^{\varepsilon} \perp_{W} y \text { if and only if } \sqrt{2} \frac{1-\varepsilon}{1+\varepsilon}\|x\|\|y\| \leq\|\| y\|x+\| x\|y\| \leq \sqrt{2} \frac{1+\varepsilon}{1-\varepsilon}\|x\|\|y\| .
$$

We study class of linear mappings preserving the approximately bisectrix-orthogonality ${ }^{\varepsilon_{W}}$. In particular, we show that if $T: \mathcal{X} \rightarrow \mathcal{Y}$ is an approximate linear similarity, then

$$
x^{\delta} \perp_{W} y \Longrightarrow T x^{\theta} \perp_{W} T y \quad(x, y \in \mathcal{X})
$$

for any $\delta \in[0,1)$ and certain $\theta \geq 0$.
\end{abstract}

\section{INTRODUCTION AND PRELIMINARIES}

There are several concepts of orthogonality appeared in the literature during the past century such as Birkhoff--James, Phythagorean, isosceles, Singer, Roberts, Diminnie, Carlsson, Rätz, $\rho$-orthogonality, area orthogonality, etc, in an arbitrary real normed space $\mathcal{X}$, which can be regarded as generalizations of orthogonality in the inner product spaces, in general [1,2]. These are of intrinsic geometric interest and have been studied by many mathematicians. Among them we recall the following ones:

(i) Birkhoff-James $\perp_{B}: x \perp_{B} y$ if $\|x\| \leq\|x+t y\|$ for all scalars $t$ (see [3]).

(ii) Phythagorean $\perp_{P}: x \perp_{P} y$ if $\|x+y\|^{2}=\|x\|^{2}+\|y\|^{2}$ (see [9]).

(iii) Isosceles $\perp_{I}: x \perp_{I} y$ if $\|x+y\|=\|x-y\|$ (see $[9,14]$ ).

(iv) Roberts $\perp_{R}: x \perp_{R} y$ if $\|x+t y\|=\|x-t y\|$ for all scalars $t$ (see [15]). The following mapping $\langle. \mid\rangle_{g}: \mathcal{X} \times \mathcal{X} \longrightarrow \mathbb{R}$ was introduced by Miličič [12]:

$$
\langle y \mid x\rangle_{g}=\frac{1}{2}\left(\rho_{+}^{\prime}(x, y)+\rho_{-}^{\prime}(x, y)\right),
$$

where mappings $\rho_{+}^{\prime}, \rho_{-}^{\prime}: \mathcal{X} \times \mathcal{X} \longrightarrow \mathbb{R}$ are defined by

$$
\rho_{ \pm}^{\prime}(x, y)=\lim _{t \rightarrow 0^{ \pm}} \frac{\|x+t y\|^{2}-\|x\|^{2}}{2 t} .
$$

In addition the $\rho$-orthogonality $x \perp_{\rho} y$ means $\langle y \mid x\rangle_{g}=0$.

Note that $\perp_{R}, \perp_{\rho} \subseteq \perp_{B}$ [1] and the relations $\perp_{P}, \perp_{I}, \perp_{B}$ are, however, independent. If $(\mathcal{H},\langle. \mid\rangle$.$) is a real inner product space, then all above relations coincide with usual$ orthogonality $\perp$ derived from $\langle. \mid\rangle.[2]$.

In the present note, we consider the so-called bisectrix-orthogonality and we study the orthogonality preserving property of this kind of orthogonality.

2010 Mathematics Subject Classification. Primary 46B20; Secondary 46C50, 47B99.

Key words and phrases. Bisectrix-orthogonality, Approximate orthogonality, Isometry, Orthogonality preserving mapping. 
Let $\mathcal{X}$ be a real normed space and $x, y \in \mathcal{X}$. The bisectrix-orthogonality relation $x \perp_{W} y$ (cf. Section 5.2 in [1]) is defined by

$$
\|\| y\|x+\| x\|y\|=\sqrt{2}\|x\|\|y\|,
$$

which for nonzero $x$ and $y$ means

$$
\left\|\frac{x}{\|x\|}+\frac{y}{\|y\|}\right\|=\sqrt{2}
$$

For instance consider the space $\left(\mathbb{R}^{2},\||\cdot|\| \mid\right)$ where $\left\||(r, s) \||=\max \{|r|,|s|\}\right.$ for $(r, s) \in \mathbb{R}^{2}$. Then $(1,0) \perp_{W}(r, s)$ if and only if either $(r, s)$ is the zero vector or

$$
(r, s) \in(\{-\sqrt{2}-1, \sqrt{2}-1\}) \times[-\sqrt{2}, \sqrt{2}] \cup([-\sqrt{2}-1, \sqrt{2}-1] \times\{-\sqrt{2}, \sqrt{2}\}) .
$$

Now we recall some properties of bisectrix-orthogonality (the proofs can be found in [1, Proposition 5.2.1]):

(P.1) $x \perp_{W} 0,0 \perp_{W} y$ for all $x, y \in \mathcal{X}$;

(P.2) $x \perp_{W} y$ if and only if $y \perp_{W} x$;

(P.3) If $x \perp_{W} y$ and $x, y \neq 0$, then $x, y$ are linearly independent;

(P.4) If $x \perp_{W} y$ and $\alpha \beta \geq 0$, then $\alpha x \perp_{W} \beta y$;

(P.5) In an inner product space, $x \perp_{W} y$ if and only if $\langle x \mid y\rangle=0$.

By the definition of bisectrix-orthogonality and Pythagorean orthogonality one can easily get the following properties:

(P.6) For all nonzero vectors $x, y \in \mathcal{X}, x \perp_{W} y$ if and only if $\frac{x}{\|x\|} \perp_{P} \frac{y}{\|y\|}$;

(P.7) For all $x, y \in S_{\mathcal{X}}=\{z \in \mathcal{X}:\|z\|=1\}, x \perp_{W} y$ if and only if $x \perp_{P} y$.

We state some relations between bisectrix-orthogonality and other orthogonalities. It is known $[1,2]$ that each of the following properties implies that the norm $\|\cdot\|$ comes from an inner product.

(P.8) $\perp_{W} \subseteq \perp_{I}$ over $\mathcal{X}$

(P.9) $\perp_{I} \subseteq \perp_{W}$ over $\mathcal{X}$

(P.10) $\perp_{W} \subseteq \perp_{B}$ over $S_{\mathcal{X}}$;

(P.11) $\perp_{B} \subseteq \perp_{W}$ over $S_{\mathcal{X}}$;

(P.12) If for all $x, y \in \mathcal{X}, x \perp_{W} y$ implies $\|x+y\|^{2}+\|x-y\|^{2} \sim 2\|x\|^{2}+2\|y\|^{2}$

where $\sim$ stands either for $\leq$ or $\geq$. An easy consequence of $\perp_{R}, \perp_{\rho} \subseteq \perp_{B}$ states that if for all $x, y \in S_{\mathcal{X}}$, the relation $x \perp_{W} y$ implies $x \perp_{R} y$ or $x \perp_{\rho} y$, then the norm $\|$. comes from an inner product.

In this paper we introduce two notions of approximate bisectrix-orthogonality ${ }^{\varepsilon_{\perp}}{ }_{W}$ and $\perp_{W}^{\varepsilon}$ in a real normed space $\mathcal{X}$ and study the class of linear mappings which preserve the approximately bisectrix-orthogonality of type ${ }^{\varepsilon} \perp_{W}$. In particular, we show that if $T: \mathcal{X} \rightarrow \mathcal{Y}$ is an approximate linear similarity, then

$$
x^{\delta} \perp_{W} y \Longrightarrow T x^{\theta} \perp_{W} T y \quad(x, y \in \mathcal{X})
$$

for any $\delta \in[0,1)$ and certain $\theta \geq 0$.

\section{Approximately BiseCtriX-ORThogonality PRESERVING MAPPINGS}

Let $\zeta, \eta$ be elements of an inner product space $(\mathcal{H},\langle. \mid\rangle$.$) and \varepsilon \in[0,1)$. The approximate orthogonality $\zeta \perp^{\varepsilon} \eta$ defined by

$$
|\langle\zeta \mid \eta\rangle| \leq \varepsilon\|\zeta\|\|\eta\|
$$

or equivalently,

$$
|\cos (\zeta, \eta)| \leq \varepsilon \quad(\zeta, \eta \neq 0) .
$$


So, it is natural to generalize the notion of approximate orthogonality for a real normed space $\mathcal{X}$. This fact motivated Chmieliński and Wójcik [5] to give for two elements $x, y \in \mathcal{X}$ the following definition of the approximate isosceles-orthogonality $x^{\varepsilon_{\perp}} \perp_{I} y$ as follows:

$$
|\|x+y\|-\|x-y\|| \leq \varepsilon(\|x+y\|+\|x-y\|) .
$$

They also introduced another approximate isosceles-orthogonality $x \perp_{I}^{\varepsilon}$ y by

$$
\left|\|x+y\|^{2}-\|x-y\|^{2}\right| \leq 4 \varepsilon\|x\|\|y\| .
$$

Inspired by the above approximate isosceles-orthogonality, we propose two definitions of approximate bisectrix-orthogonality.

Let $\varepsilon \in[0,1)$ and $x, y \in \mathcal{X}$, let us put $x^{\varepsilon} \perp_{W} y$ if

$$
\|\| y\|x+\| x\|y\|-\sqrt{2}\|x\|\|y\| \mid \leq \varepsilon(\|\| y\|x+\| x\|y\|+\sqrt{2}\|x\|\|y\|)
$$

or equivalently,

$$
\sqrt{2} \frac{1-\varepsilon}{1+\varepsilon}\|x\|\|y\| \leq\|\| y\|x+\| x\|y\| \leq \sqrt{2} \frac{1+\varepsilon}{1-\varepsilon}\|x\|\|y\|,
$$

which means

$$
\sqrt{2} \frac{1-\varepsilon}{1+\varepsilon} \leq\left\|\frac{x}{\|x\|}+\frac{y}{\|y\|}\right\| \leq \sqrt{2} \frac{1+\varepsilon}{1-\varepsilon}
$$

for nonzero vectors $x$ and $y$.

Also we define $x \perp_{W}^{\varepsilon} y$ if

$$
\|\| y\|x+\| x\|y\|^{2}-2\|x\|^{2}\|y\|^{2} \mid \leq 2 \varepsilon\|x\|^{2}\|y\|^{2}
$$

or equivalently,

$$
\sqrt{2(1-\varepsilon)}\|x\|\|y\| \leq\|\| y\|x+\| x\|y\| \leq \sqrt{2(1+\varepsilon)}\|x\|\|y\|,
$$

which means

$$
\sqrt{2(1-\varepsilon)} \leq\left\|\frac{x}{\|x\|}+\frac{y}{\|y\|}\right\| \leq \sqrt{2(1+\varepsilon)}
$$

for nonzero $x, y \in \mathcal{X}$.

It is easy to check that in the case where the norm comes from a real-valued inner product we have

$$
x^{\varepsilon} \perp_{W} y \Leftrightarrow|\langle x, y\rangle| \leq \frac{4 \varepsilon}{(1-\varepsilon)^{2}}\|x\|\|y\|
$$

and

$$
x \perp_{W}^{\varepsilon} y \Leftrightarrow|\langle x, y\rangle| \leq \varepsilon\|x\|\|y\| \Leftrightarrow x \perp^{\varepsilon} y
$$

Thus the second approximate bisectrix-orthogonality coincides with the natural notion of approximate orthogonality for inner product spaces.

Note that the relations ${ }^{\varepsilon} \perp_{W}$ and $\perp_{W}^{\varepsilon}$ are symmetric and almost homogeneous in the sense that

$$
x^{\varepsilon} \perp_{W} y \Longrightarrow y^{\varepsilon} \perp_{W} x \text { and } \alpha x^{\varepsilon} \perp_{W} \beta y \text { for } \alpha \beta \geq 0
$$

and

$$
x \perp_{W}^{\varepsilon} y \Longrightarrow y \perp_{W}^{\varepsilon} x \text { and } \alpha x \perp_{W}^{\varepsilon} \beta y \text { for } \alpha \beta \geq 0 \text {. }
$$


Remark 2.1. It is easy to see that $\perp_{W}^{\varepsilon}$ implies ${ }^{\varepsilon} \perp_{W}$ with the same $\varepsilon$. Also if $\varepsilon \in\left[0, \frac{1}{16}\right)$, then ${ }^{\varepsilon_{W}}{ }_{W}$ implies $\perp_{W}^{16 \varepsilon}$. Indeed, for $x, y \neq 0$, since $0 \leq \varepsilon<\frac{1}{16}$, so $\frac{1+\varepsilon}{1-\varepsilon} \leq \sqrt{1+16 \varepsilon}$ and $\sqrt{1-16 \varepsilon} \leq \frac{1-\varepsilon}{1+\varepsilon}$. Hence $x^{\varepsilon} \perp_{W} y$ implies

$$
\sqrt{2(1-16 \varepsilon)} \leq\left\|\frac{x}{\|x\|}+\frac{y}{\|y\|}\right\| \leq \sqrt{2(1+16 \varepsilon)}
$$

or equivalently, $x \perp_{W}^{16 \varepsilon} y$.

Now, suppose that $\mathcal{X}$ and $\mathcal{Y}$ are real normed spaces of dimensions greater than or equal to two and let $\delta, \varepsilon \in[0,1)$. We say that a linear mapping $T: \mathcal{X} \rightarrow \mathcal{Y}$ preserves the approximate bisectrix-orthogonality if

$$
x^{\delta} \perp_{W} y \Longrightarrow T x^{\varepsilon} \perp_{W} T y \quad(x, y \in \mathcal{X}) .
$$

Notice that if $\delta=\varepsilon=0$, we have

$$
x \perp_{W} y \Longrightarrow T x \perp_{W} T y \quad(x, y \in \mathcal{X}),
$$

and we say that $T$ preserves the bisectrix-orthogonality.

Koldobsky [10] (for real spaces) and Blanco and Turnšek [4] (for real and complex ones) proved that a linear mapping $T: \mathcal{X} \rightarrow \mathcal{Y}$ preserving the Birkhoff orthogonality has to be a similarity, i.e., a non-zero-scalar multiple of an isometry. Further, Chmieliński and Wójcik [7, 16] proved that a linear mapping $T: \mathcal{X} \rightarrow \mathcal{Y}$ preserving the $\rho$-orthogonality has to be a similarity. Approximately orthogonality preserving mappings in the framework of normed spaces have been recently studied. In the case where $\delta=0$, Mojškerc and Turnšek [13] and Chmieliński [6] verified the properties of mappings that preserve approximate Birkhoff orthogonality. Also Chmieliński and Wójcik [5, 7] studied some properties of mappings that preserve approximate isosceles-orthogonality and $\rho$ orthogonality in the case when $\delta=0$. Recently Zamani and Moslehian [17] studied approximate Roberts orthogonality preserving mappings.

The next lemma plays an essential role in our work. It provides indeed a reverse of the triangle inequality; see [8].

Lemma 2.2. [11, Theorem 1] Let $\mathcal{X}$ be a normed space and $x, y \in \mathcal{X} \backslash\{0\}$. Then

$$
\begin{aligned}
\|x\|+\|y\|+\left(\| \frac{x}{\|x\|}\right. & \left.+\frac{y}{\|y\|} \|-2\right) \max \{\|x\|,\|y\|\} \\
& \leq\|x+y\| \\
& \leq\|x\|+\|y\|+\left(\left\|\frac{x}{\|x\|}+\frac{y}{\|y\|}\right\|-2\right) \min \{\|x\|,\|y\|\}
\end{aligned}
$$

To reach our main result, we need some lemmas, which are interesting on their own right. We state some prerequisites for the first lemma. For a bounded linear mapping $T: \mathcal{X} \rightarrow \mathcal{Y}$, let $\|T\|=\sup \{\|T x\| ;\|x\|=1\}$ denote the operator norm and $[T]:=$ $\inf \{\|T x\| ;\|x\|=1\}$. Notice that for any $x \in \mathcal{X}$, we have $[T]\|x\| \leq\|T x\| \leq\|T\|\|x\|$.

Lemma 2.3. Let $\delta, \varepsilon \in[0,1)$. If a nonzero bounded linear mapping $T: \mathcal{X} \rightarrow \mathcal{Y}$ satisfies

$$
\frac{1-\varepsilon}{1+\varepsilon} \gamma\|x\| \leq\|T x\| \leq \frac{1+\varepsilon}{1-\varepsilon} \gamma\|x\|
$$

for all $x \in \mathcal{X}$ and all $\gamma \in\left[\frac{1-\delta}{1+\delta}[T], \frac{1+\delta}{1-\delta}\|T\|\right]$, then

$$
x^{\delta} \perp_{W} y \Longrightarrow T x^{\varepsilon} \perp_{W} T y \quad(x, y \in \mathcal{X}) .
$$


Proof. Let $x, y \in \mathcal{X} \backslash\{0\}$ and $x^{\delta} \perp_{W} y$. Then $\sqrt{2} \frac{1-\delta}{1+\delta} \leq\left\|\frac{x}{\|x\|}+\frac{y}{\|y\|}\right\| \leq \sqrt{2} \frac{1+\delta}{1-\delta}$. If $x=s y$ for some $s \in \mathbb{R} \backslash\{0\}$, then for $\gamma=\frac{1-\delta}{1+\delta}[T]$ we have

$$
\begin{aligned}
& \left\|\frac{T x}{\|T x\|}+\frac{T y}{\|T y\|}\right\|=\left\|T\left(\frac{x}{\|T x\|}+\frac{y}{\|T y\|}\right)\right\| \\
& =\left\|T\left(\frac{s y}{\|s T y\|}+\frac{y}{\|T y\|}\right)\right\| \\
& =\frac{\|y\|}{\|T y\|}\left\|T\left(\frac{s y}{\|s y\|}+\frac{y}{\|y\|}\right)\right\| \\
& =\frac{\|y\|}{\|T y\|}\left\|T\left(\frac{x}{\|x\|}+\frac{y}{\|y\|}\right)\right\| \\
& \leq \frac{\|y\|}{\|T y\|} \frac{1+\varepsilon}{1-\varepsilon} \gamma\left\|\frac{x}{\|x\|}+\frac{y}{\|y\|}\right\| \\
& =\frac{\|y\|}{\|T y\|} \frac{1+\varepsilon}{1-\varepsilon} \frac{1-\delta}{1+\delta}[T]\left\|\frac{x}{\|x\|}+\frac{y}{\|y\|}\right\| \\
& \leq \frac{1+\varepsilon}{1-\varepsilon} \frac{1-\delta}{1+\delta} \sqrt{2} \frac{1+\delta}{1-\delta} \\
& =\sqrt{2} \frac{1+\varepsilon}{1-\varepsilon},
\end{aligned}
$$

whence $\left\|\frac{T x}{\|T x\|}+\frac{T y}{\|T y\|}\right\| \leq \sqrt{2} \frac{1+\varepsilon}{1-\varepsilon}$. Similarly, $\sqrt{2} \frac{1-\varepsilon}{1+\varepsilon} \leq\left\|\frac{T x}{\|T x\|}+\frac{T y}{\|T y\|}\right\|$. Thus $T x^{\varepsilon} \perp_{W} T y$. Assume that $x, y$ are linearly independent. Set $\gamma_{0}:=\frac{\sqrt{2}}{\left\|\frac{x}{\|T x\|}+\frac{y}{\|T y\|}\right\|}$. We may assume that $\frac{\|x\|}{\|T x\|} \leq \frac{\|y\|}{\|T y\|}$. By Lemma 2.2 we have

$$
\begin{aligned}
\left\|\frac{x}{\|T x\|}+\frac{y}{\|T y\|}\right\| & \leq \frac{\|x\|}{\|T x\|}+\frac{\|y\|}{\|T y\|}+\left(\left\|\frac{x}{\|x\|}+\frac{y}{\|y\|}\right\|-2\right) \min \left\{\frac{\|x\|}{\|T x\|}, \frac{\|y\|}{\|T y\|}\right\} \\
& \leq \frac{\|y\|}{\|T y\|}+\left(\sqrt{2} \frac{1+\delta}{1-\delta}-1\right) \frac{\|x\|}{\|T x\|} \\
& \leq \frac{1}{[T]}+\left(\sqrt{2} \frac{1+\delta}{1-\delta}-1\right) \frac{1}{[T]} \\
& =\sqrt{2} \frac{1+\delta}{1-\delta} \frac{1}{[T]} .
\end{aligned}
$$

So that $\gamma_{0} \geq \frac{\sqrt{2}}{\sqrt{2} \frac{1+\delta}{1-\delta} \frac{1}{[T]}}=\frac{1-\delta}{1+\delta}[T]$.

Similarly we get $\gamma_{0} \leq \frac{1+\delta}{1-\delta}\|T\|$. Thus $\gamma_{0} \in\left[\frac{1-\delta}{1+\delta}[T], \frac{1+\delta}{1-\delta}\|T\|\right]$. Our hypothesis implies that

$$
\frac{1-\varepsilon}{1+\varepsilon} \gamma_{0}\|z\| \leq\|T z\| \leq \frac{1+\varepsilon}{1-\varepsilon} \gamma_{0}\|z\| \quad(z \in \mathcal{X})
$$

or equivalently,

$$
\left|\|T z\|-\gamma_{0}\|z\|\right| \leq \varepsilon\left(\|T z\|+\gamma_{0}\|z\|\right) \quad(z \in \mathcal{X})
$$


Putting $\|T y\| x+\|T x\| y$ instead of $z$ in the above inequality we get

$$
\begin{aligned}
& \|\|\|T y\| T x+\|T x\| T y\left\|-\frac{\sqrt{2}}{\left\|\frac{x}{\|T x\|}+\frac{y}{\|T y\|}\right\|}\right\|\|T y\| x+\|T x\| y\|\| \\
& \leq \varepsilon\left(\|\| T y\|T x+\| T x\|T y\|+\frac{\sqrt{2}}{\left\|\frac{x}{\|T x\|}+\frac{y}{\|T y\|}\right\|}\|\| T y\|x+\| T x\|y\|\right) .
\end{aligned}
$$

Thus

$|\|\| T y\|T x+\| T x\|T y\|-\sqrt{2}\|T x\|\|T y\|| \leq \varepsilon(\|\| T y\|T x+\| T x\|T y\|+\sqrt{2}\|T x\|\|T y\|)$, whence $T x^{\varepsilon} \perp_{W} T y$.

Lemma 2.4. Let $\delta, \varepsilon \in[0,1)$. If a nonzero bounded linear mapping $T: \mathcal{X} \rightarrow \mathcal{Y}$ satisfies $\frac{1+\delta}{1-\delta}\|T z\|\|u\| \leq \frac{1+\varepsilon}{1-\varepsilon}\|T u\|\|z\|$ for all $z, u \in \mathcal{X}$, then

$$
x^{\delta} \perp_{W} y \Longrightarrow T x^{\varepsilon} \perp_{W} T y \quad(x, y \in \mathcal{X}) .
$$

Proof. By our assumption we have, $\frac{1+\delta}{1-\delta}\|T z\| \leq \frac{1+\varepsilon}{1-\varepsilon}\|T u\|$ for all $z, u$ with $\|z\|=\|u\|=1$. Passing to the infimum over $\|u\|=1$, we get

$$
\frac{1+\delta}{1-\delta}\|T z\| \leq \frac{1+\varepsilon}{1-\varepsilon}[T] \quad(\|z\|=1)
$$

and passing to the supremum over $\|z\|=1$ we obtain

$$
\frac{1+\delta}{1-\delta}\|T\| \leq \frac{1+\varepsilon}{1-\varepsilon}[T]
$$

Now, let $\gamma \in\left[\frac{1-\delta}{1+\delta}[T], \frac{1+\delta}{1-\delta}\|T\|\right]$ and $x \in \mathcal{X}$. Therefore we have

$$
\begin{aligned}
\frac{1-\varepsilon}{1+\varepsilon} \gamma\|x\| & \leq \frac{1-\varepsilon}{1+\varepsilon} \times \frac{1+\delta}{1-\delta}\|T\|\|x\| \\
& \leq \frac{1-\varepsilon}{1+\varepsilon} \times \frac{1+\delta}{1-\delta} \times \frac{1+\varepsilon}{1-\varepsilon} \times \frac{1-\delta}{1+\delta}[T]\|x\| \\
& \leq\|T x\| \\
& \leq\|T\|\|x\| \\
& \leq \frac{1+\varepsilon}{1-\varepsilon} \times \frac{1-\delta}{1+\delta}[T]\|x\| \\
& \leq \frac{1+\varepsilon}{1-\varepsilon} \times \frac{1-\delta}{1+\delta} \times \frac{1+\delta}{1-\delta} \gamma\|x\| \\
& =\frac{1+\varepsilon}{1-\varepsilon} \gamma\|x\| .
\end{aligned}
$$

Thus

$$
\frac{1-\varepsilon}{1+\varepsilon} \gamma\|x\| \leq\|T x\| \leq \frac{1+\varepsilon}{1-\varepsilon} \gamma\|x\|
$$

Making a use of Lemma 2.3 just completes the proof.

We are now in position to establish the main result. Following [13], we say that a linear mapping $U: \mathcal{X} \rightarrow \mathcal{Y}$ is an approximate linear isometry if

$$
\left(1-\varphi_{1}(\varepsilon)\right)\|z\| \leq\|U z\| \leq\left(1+\varphi_{2}(\varepsilon)\right)\|z\| \quad(z \in \mathcal{X}),
$$

where $\varphi_{1}(\varepsilon) \rightarrow 0$ and $\varphi_{2}(\varepsilon) \rightarrow 0$ as $\varepsilon \rightarrow 0$. Notice that if $\varphi_{1}(\varepsilon)=\varphi_{2}(\varepsilon)=0$, then $U$ is an isometry. 
A linear mapping $U: \mathcal{X} \rightarrow \mathcal{Y}$ is said to be an approximate similarity if it is a non-zero-scalar multiple of an approximate linear isometry, or equivalently it satisfies

$$
\lambda\left(1-\varphi_{1}(\varepsilon)\right)\|w\| \leq\|U w\| \leq \lambda\left(1+\varphi_{2}(\varepsilon)\right)\|w\|
$$

for some unitary $U$, some $\lambda>0$ and for all $w \in \mathcal{X}$, where $\varphi_{1}(\varepsilon) \rightarrow 0$ and $\varphi_{2}(\varepsilon) \rightarrow 0$ as $\varepsilon \rightarrow 0$.

Theorem 2.5. Let $U: \mathcal{X} \rightarrow \mathcal{Y}$ be an approximate linear similarity and $\delta \in[0,1)$. If a nonzero bounded linear mapping $T: \mathcal{X} \rightarrow \mathcal{Y}$ satisfies $\|T-U\| \leq \varepsilon\|U\|$, then

$$
x^{\delta} \perp_{W} y \Longrightarrow T x^{\theta} \perp_{W} T y \quad(x, y \in \mathcal{X}),
$$

where $\theta=\frac{2 \delta+2 \varepsilon+(1-\delta) \varphi_{1}(\varepsilon)+(1+\delta+2 \varepsilon) \varphi_{2}(\varepsilon)}{2+2 \delta \varepsilon-(1-\delta) \varphi_{1}(\varepsilon)+(1+\delta+2 \delta \varepsilon) \varphi_{2}(\varepsilon)}$.

Proof. For any $w \in \mathcal{X}$ we have

$$
|\|T w\|-\|U w\|| \leq\|T w-U w\| \leq\|T-U\|\|w\| \leq \varepsilon\|U\|\|w\| \leq \varepsilon \lambda\left(1+\varphi_{2}(\varepsilon)\right)\|w\|,
$$

whence

$$
-\varepsilon \lambda\left(1+\varphi_{2}(\varepsilon)\right)\|w\| \leq\|T w\|-\|U w\| \leq \varepsilon \lambda\left(1+\varphi_{2}(\varepsilon)\right)\|w\|
$$

Since

$$
\lambda\left(1-\varphi_{1}(\varepsilon)\right)\|w\| \leq\|U w\| \leq \lambda\left(1+\varphi_{2}(\varepsilon)\right)\|w\|,
$$

therefore we get

$$
\lambda\left[\left(1-\varphi_{1}(\varepsilon)\right)-\varepsilon\left(1+\varphi_{2}(\varepsilon)\right)\right]\|w\| \leq\|T w\| \leq \lambda(1+\varepsilon)\left(1+\varphi_{2}(\varepsilon)\right)\|w\| .
$$

Thus for any $z, u \in \mathcal{X}$, we have

$$
\begin{aligned}
\frac{1+\delta}{1-\delta}\|T z\|\|u\| & \leq \frac{1+\delta}{1-\delta} \lambda(1+\varepsilon)\left(1+\varphi_{2}(\varepsilon)\right)\|z\| \frac{\|T u\|}{\lambda\left[\left(1-\varphi_{1}(\varepsilon)\right)-\varepsilon\left(1+\varphi_{2}(\varepsilon)\right)\right]} \\
& =\frac{(1+\varepsilon)\left(1+\varphi_{2}(\varepsilon)\right)(1+\delta)}{\left[\left(1-\varphi_{1}(\varepsilon)\right)-\varepsilon\left(1+\varphi_{2}(\varepsilon)\right)\right](1-\delta)}\|T u\|\|z\| \\
& =\frac{1+\frac{2 \delta+2 \varepsilon+(1-\delta) \varphi_{1}(\varepsilon)+(1+\delta+2 \varepsilon) \varphi_{2}(\varepsilon)}{2+2 \delta \varepsilon-(1-\delta) \varphi_{1}(\varepsilon)+(1+\delta+2 \delta \varepsilon) \varphi_{2}(\varepsilon)}}{1-\frac{2 \delta+2 \varepsilon+(1-\delta) \varphi_{1}(\varepsilon)+(1+\delta+2 \varepsilon) \varphi_{2}(\varepsilon)}{2+2 \delta \varepsilon-(1-\delta) \varphi_{1}(\varepsilon)+(1+\delta+2 \delta \varepsilon) \varphi_{2}(\varepsilon)}}\|T u\|\|z\| \\
& =\frac{1+\theta}{1-\theta}\|T u\|\|z\| .
\end{aligned}
$$

Therefore $\frac{1+\delta}{1-\delta}\|T z\|\|u\| \leq \frac{1+\theta}{1-\theta}\|T u\|\|z\|$. Now the assertion follows from Lemma 2.4.

As a consequence, with $\varepsilon=0$ and $T=U$, we have

Corollary 2.6. Let $T: \mathcal{X} \rightarrow \mathcal{Y}$ be an approximate linear similarity. Then

$$
x^{\delta} \perp_{W} y \Longrightarrow T x^{\theta} \perp_{W} T y \quad(x, y \in \mathcal{X})
$$

for any $\delta \in[0,1)$, where $\theta=\frac{2 \delta+(1-\delta) \varphi_{1}(\varepsilon)+(1+\delta) \varphi_{2}(\varepsilon)}{2-(1-\delta) \varphi_{1}(\varepsilon)+(1+\delta) \varphi_{2}(\varepsilon)}$.

Acknowledgement. The author would like to thank his PhD supervisor, Prof. M. S. Moslehian, for his useful comments improving the paper. 


\section{REFERENCES}

1. C. Alsina, J. Sikorska and M. Santos Tomás, Norm Derivatives and Characterizations of Inner Product Spaces, World Scientific, Hackensack, NJ, 2009.

2. D. Amir, Characterization of Inner Product Spaces, Birkhäuser Verlag, Basel-Boston-Stuttgart, 1986.

3. G. Birkhoff, Orthogonality in linear metric spaces, Duke Math. J. 1 (1935), 169-172.

4. A. Blanco and A. Turnšek, On maps that preserves orthogonality in normed spaces, Proc. Roy. Soc. Edinburgh Sect. A 136 (2006), 709-716.

5. J. Chmieliński and P. Wójcik, Isosceles-orthogonality preserving property and its stability, Nonlinear Anal. 72 (2010), 1445-1453.

6. J. Chmieliński, Remarks on orthogonality pereserving mappings in normed spaces and some stability problems, Banach J. Math. Anal. Bold (2007), no. 1, 117-124.

7. J. Chmieliński and P. Wójcik, On a $\rho$-orthogonality, Aequationes Math. 80 (2010), 45-55.

8. F. Dadipour, M. S. Moslehian, J. M. Rassias and S.-E. Takahasi, Characterization of a generalized triangle inequality in normed spaces, Nonlinear Anal-TMA 75 (2012), no. 2, 735-741.

9. R. C. James, Orthogonality in normed linear spaces, Duke Math. J. 12 (1945), 291-301.

10. D. Koldobsky, Operators preserving orthogonality are isometries, Proc. Roy. Soc. Edinburgh Sect. A 123 (1993), 835-837.

11. L. Maligranda, Simple norm inequalities, Amer. Math. Monthly 113 (2006), 256-260.

12. P. M. Miličić, Sur la G-orthogonalité dans les espéaceésnormés, Math. Vesnik. 39 (1987), 325-334.

13. B. Mojškerc and A. Turnšek, Mappings approximately preserving orthogonality in normed spaces, Nonlinear Anal. 73 (2010), 3821-3831.

14. M. Mirzavaziri and M. S. Moslehian, Orthogonal constant mappings in isoceles orthogonal spaces, Kragujevac J. Math. 29 (2006), 133-140.

15. B. D. Roberts, On the geometry of abstract vector spaces, Tôhoku Math. J. 39 (1934), 42-59.

16. P. Wójcik, Linear mappings preserving $\rho$-orthogonality, J. Math. Anal. Appl. 386 (2012), 171-176.

17. A. Zamani and M.S. Moslehian, Approximate Roberts orthogonality, Aequat. Math. 89 (2015), $529-541$.

Department of Pure Mathematics, Center of Excellence in Analysis on Algebraic Structures (CEAAS), Ferdowsi University of Mashhad, P.O. Box 1159, Mashhad 91775, IRAN.

E-mail address: zamani.ali85@yahoo.com. 